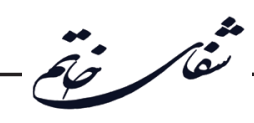

\title{
Effectiveness of Psychodrama based on Unity-oriented Therapeutic Approach on Resilience of Patients with Multiple Sclerosis
}

\author{
Saeed Dehnavi $^{{ }^{*}}$, Ali Zadeh Mohammadi ${ }^{2}$, Seyedeh Farnaz Hashemi ${ }^{3}$, Mahin Bajelan ${ }^{4}$ \\ ${ }^{1}$ Department of Clinical Psychology, Science and Research Branch, Islamic Azad University, Qazvin, Iran \\ ${ }^{2}$ Family Research Institute, Shahid Beheshti University, Tehran, Iran \\ ${ }^{3}$ Department of Psychology, Karaj Branch, Islamic Azad University, Karaj, Iran \\ ${ }^{4}$ Department of Psychology, Science and Research Branch, Islamic Azad University, Tehran, Iran
}

\begin{abstract}
Article Info:
Received: 30 Jul 2017

Revised: 25 Sep 2017

Accepted: 8 Nov 2017
\end{abstract}

\section{ABSTRACT}

Introduction: Multiple sclerosis is a demyelinating disease of the central nervous system. This disease is the most common cause of disability in young age. The purpose of this study was to investigate the effectiveness of psychodrama based on unity-oriented therapeutic approach on resilience of patients with multiple sclerosis. Materials and Methods: This study was a quasi-experimental research investigation, using pre- post and follow-up testing plan with a control group. Through a convenience sampling technique, a total of 20 individuals were selected among female patients with multiple sclerosis referring to Iranian Multiple Sclerosis Society. Then, the subjects were randomly placed into two control and experimental groups. The experimental group participated in a twelve-session unity-focused psychodrama therapy plan for 6 weeks, while the control group received no intervention. In order to collect data, the Connor-Davidson resilience scale (CD-RISC; 2003) was used. Results: The results revealed a significant difference between two groups for the post test and follow-up test scores. The psychodrama intervention based on unity-oriented therapeutic approach can be used as an effective modality to enhance a sense of resilience among patients with multiple sclerosis. Conclusion: Our findings suggest using psychodrama based on unity-oriented for improving mental condition of patients suffering from multiple sclerosis. The psychodrama based on unityoriented therapeutic approach provides the integrity and acquisition of semantic knowledge about patients' negative experiences. Furthermore, this approach can foster the acceptance of illness, as of being connected with the world and a sense of immortality, among these patients.

Key words:

1. Psychodrama

2. Multiple Sclerosis

3. Central Nervous System

*Corresponding Author: Saeed Dehnavi

E-mail: Saeed.dehnavi@ymail.com 


\title{
اثربخشى روان نمايشگرى مبتنى بر رويكرد وحدتمدار بر تابآورى بيماران مبتلا به مالتييل اسكلروز
}

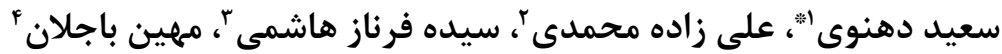 \\ اخروه روانشناسى بالينى، واحد علوم و تحقيقات، دانشخاه آزاد اسلامى، قزوين، ايران

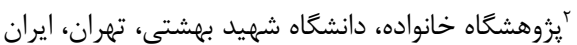

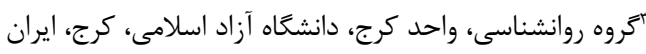

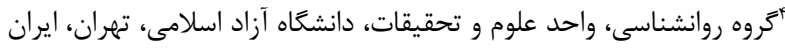

\section{اطلاعات مقاله:}

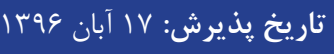

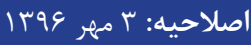

تاريخ دريافت: 1 مرداد وهبا

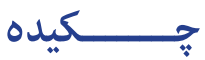

مقدمه: مالتييل اسكلروز يك بيمارى ميلينزداى سيستم عصبى مركزى است. اين بيمارى رايجترين علت

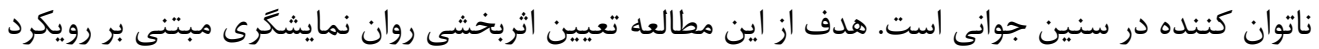

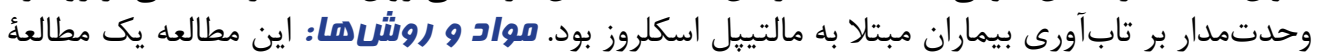

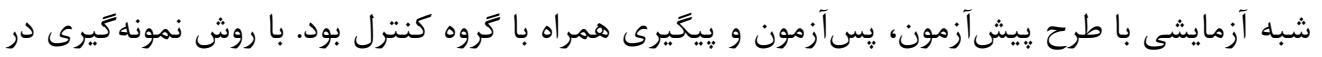

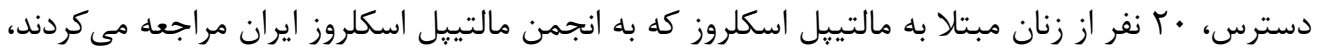

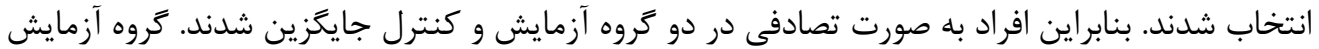

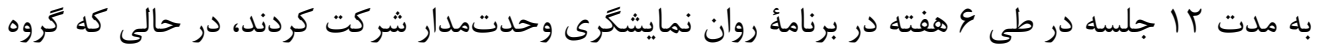

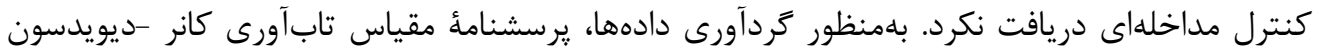

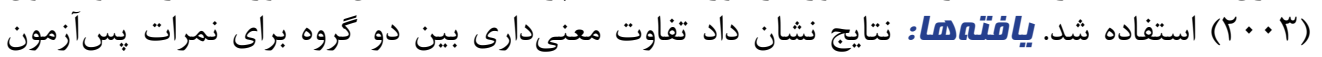

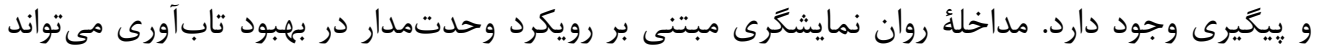

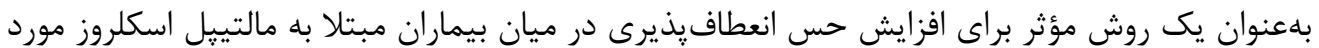

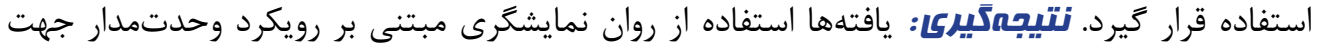

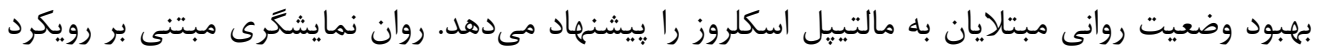

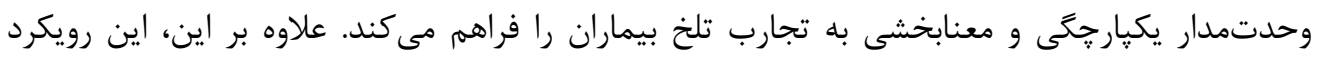

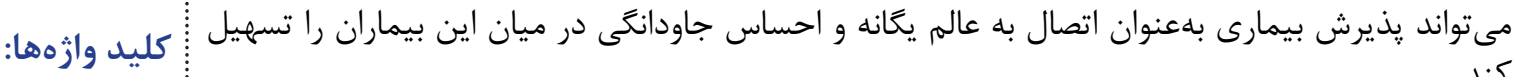

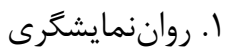

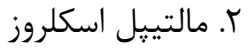
r. مركزى كند. 


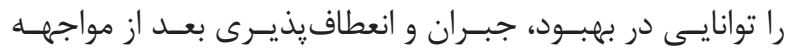

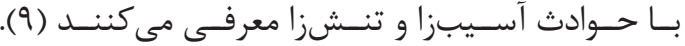

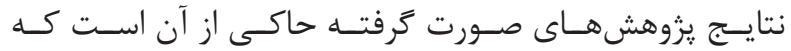

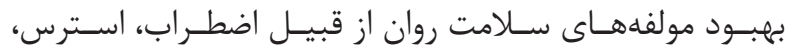

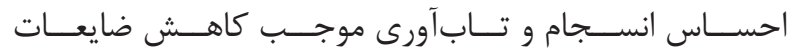

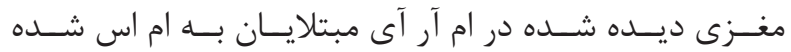

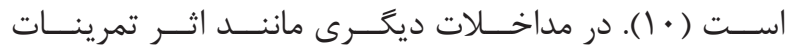

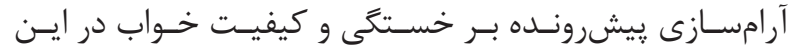

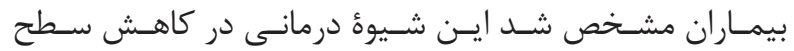

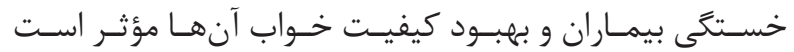

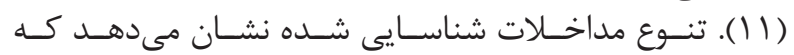

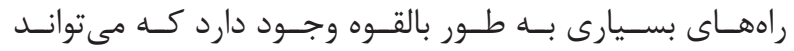

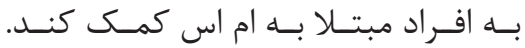

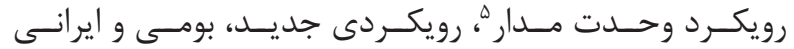

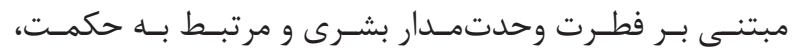

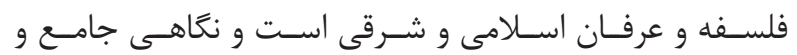

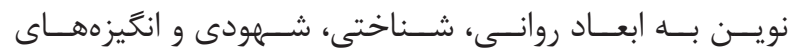

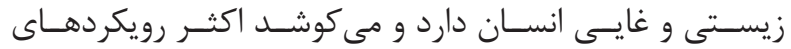

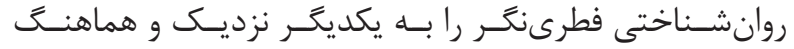

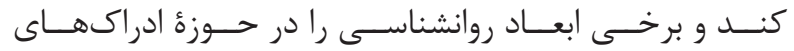

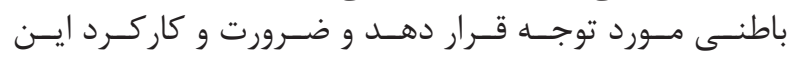

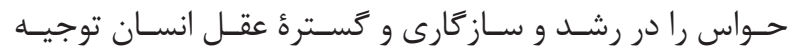

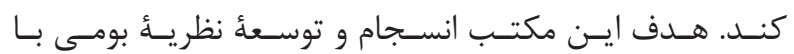

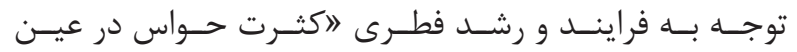

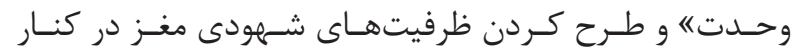

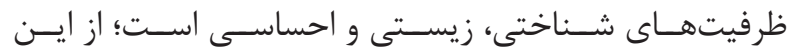

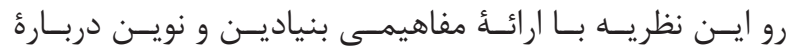

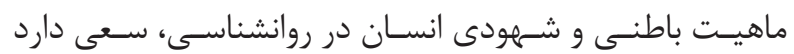

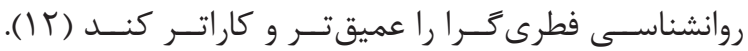

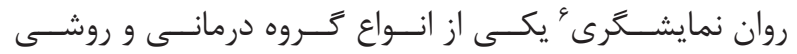

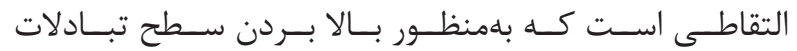

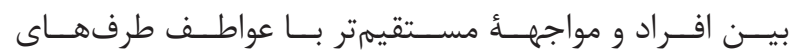

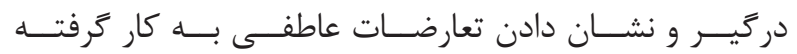

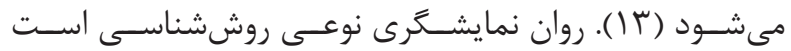

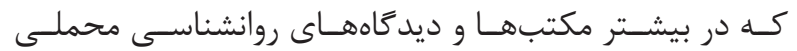

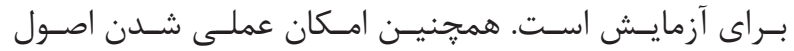

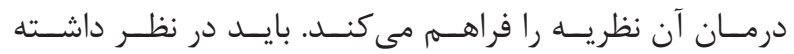

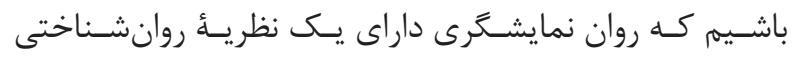

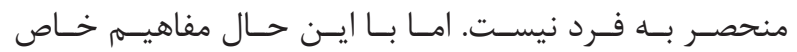

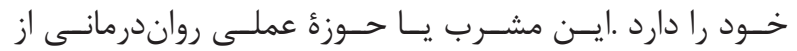

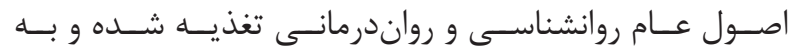

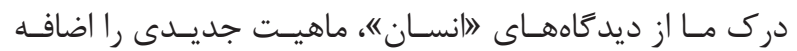

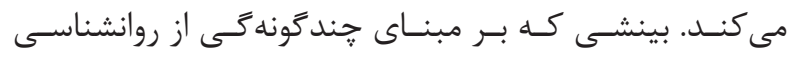

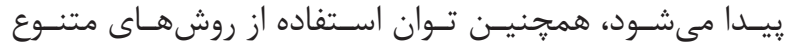

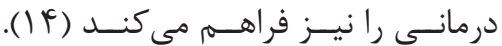

${ }^{1}$ Multiple sclerosis

${ }^{2}$ Optic nerves

${ }^{3}$ Spinal cord

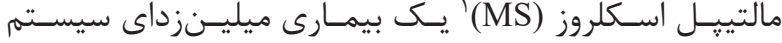

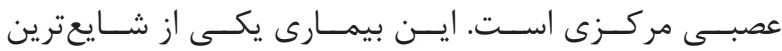

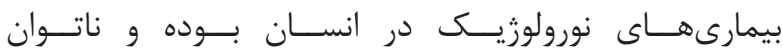

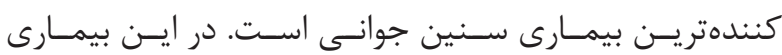

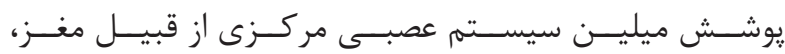

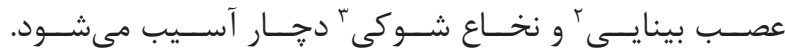

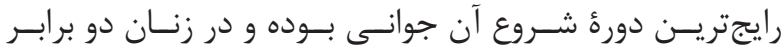

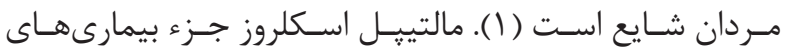

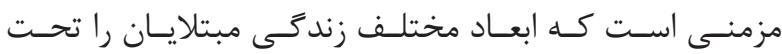

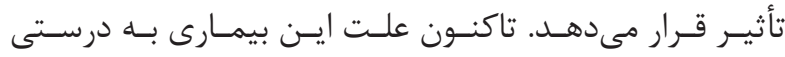

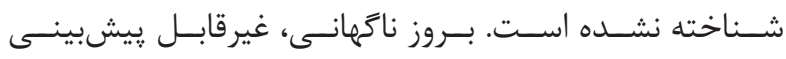

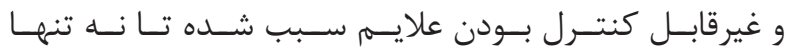

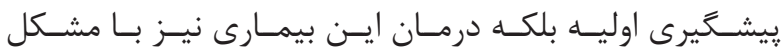

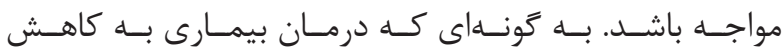

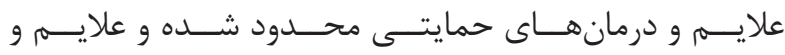

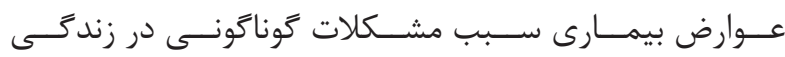

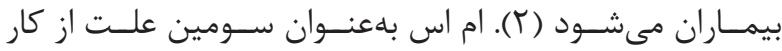

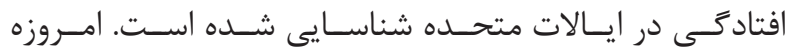

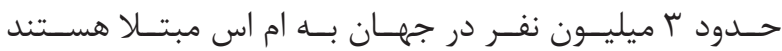

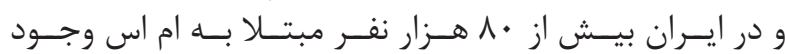

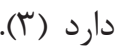

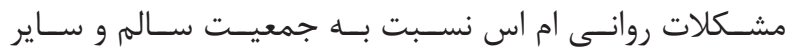

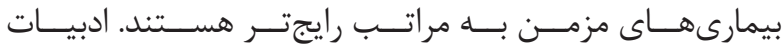

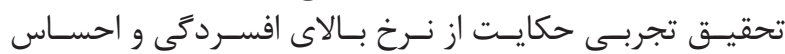

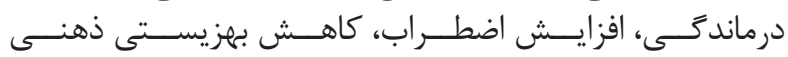

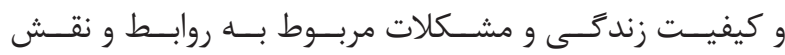

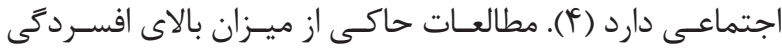

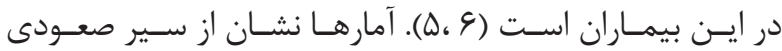

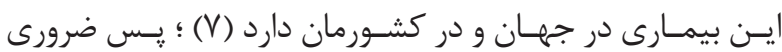

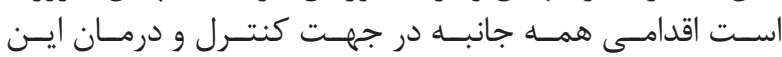
بيمـارى انجـام شـود.

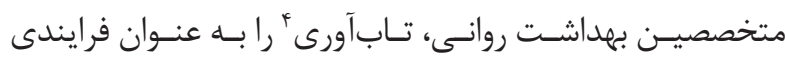

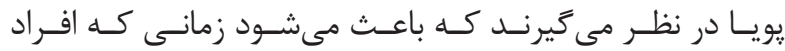

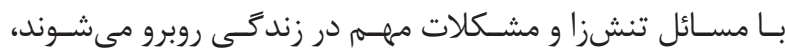

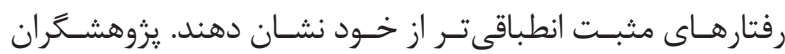

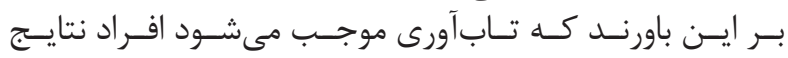

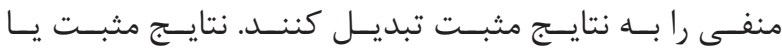

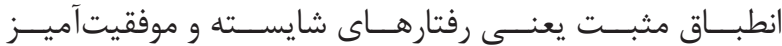

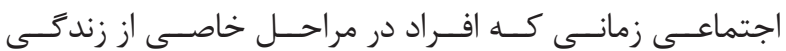

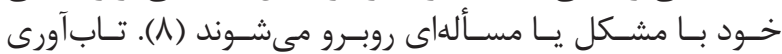

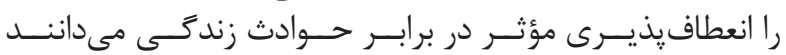

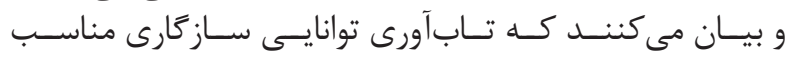

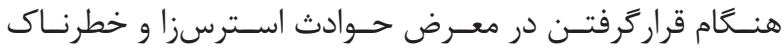

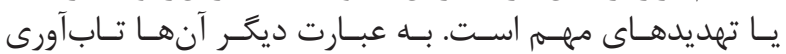

${ }^{4}$ Resilience

${ }^{5}$ Unity-oriented approach

${ }^{6}$ Psychodrama 


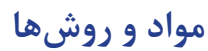

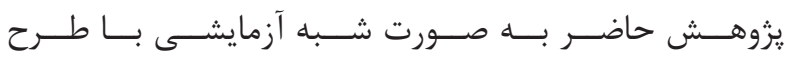

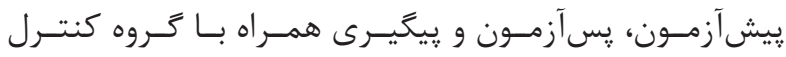

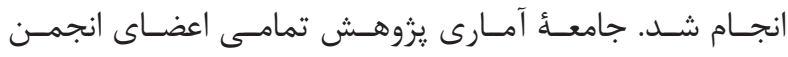

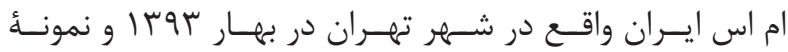

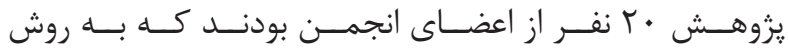

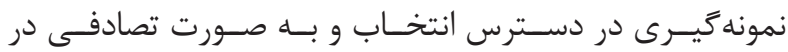

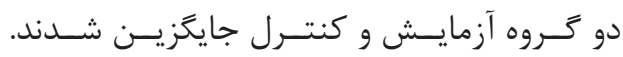

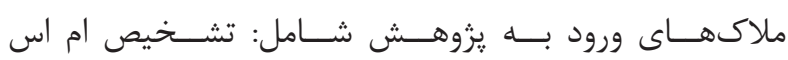

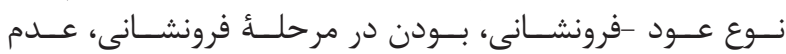

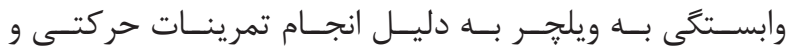

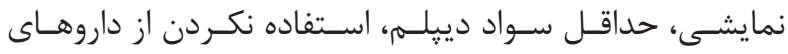

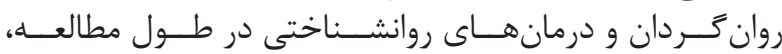

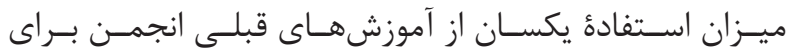

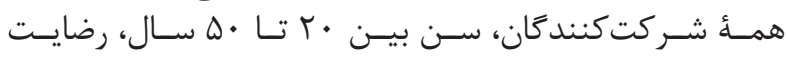

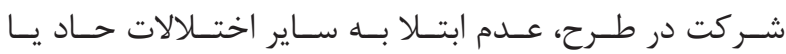

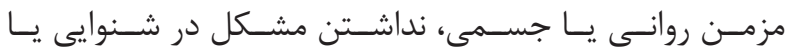

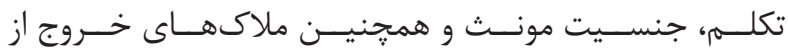

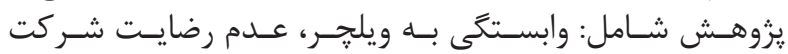

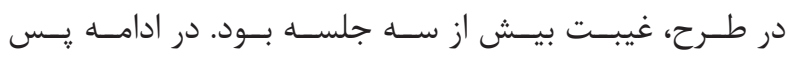

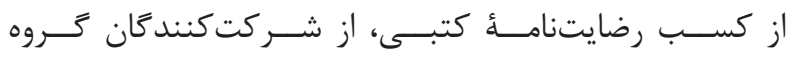

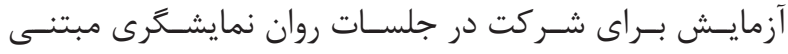

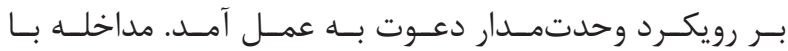

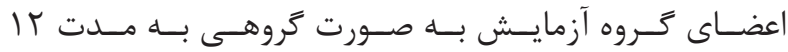

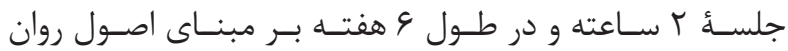

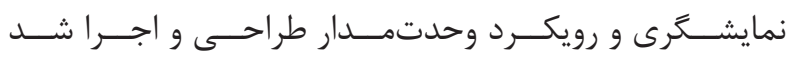

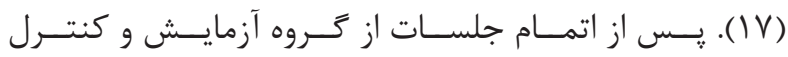

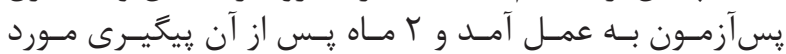

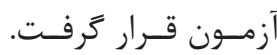

يرسشنامهُ تاب آورى كانر و ديويدسون

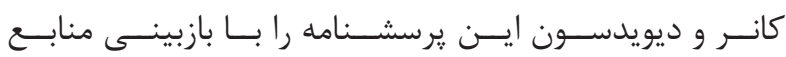

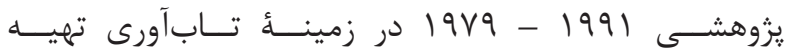

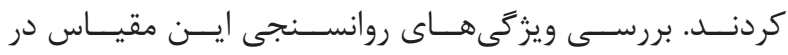

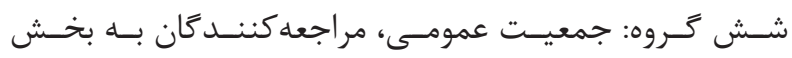

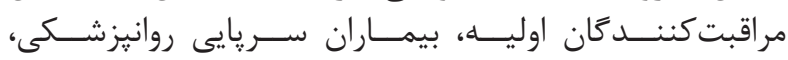

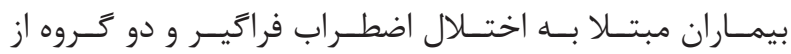

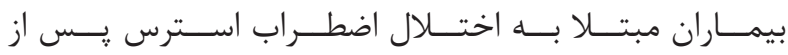

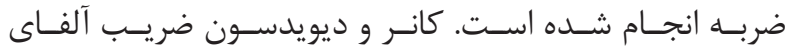

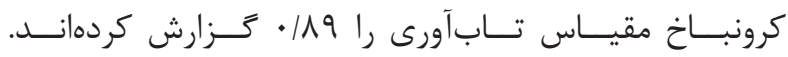

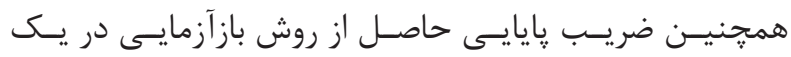

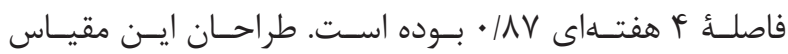

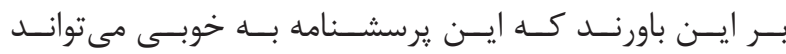

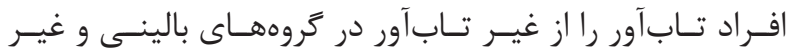

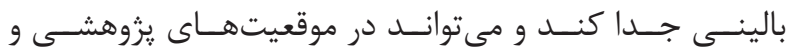

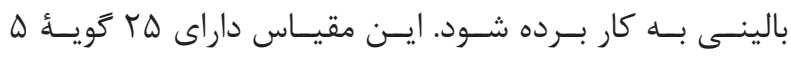

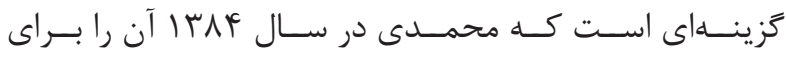

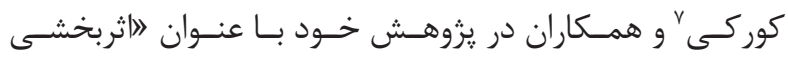

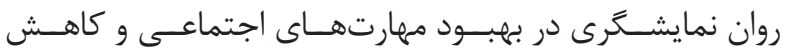

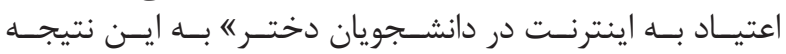

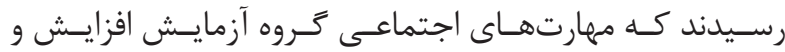

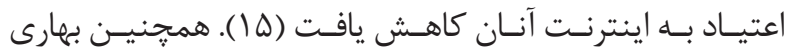

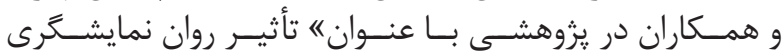

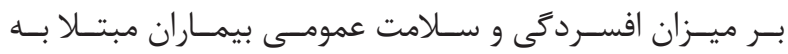

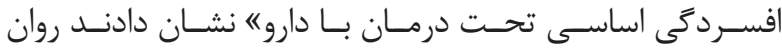

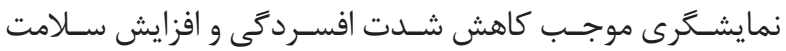

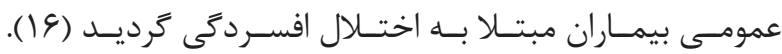

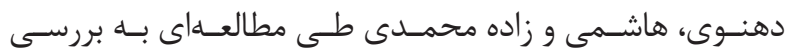

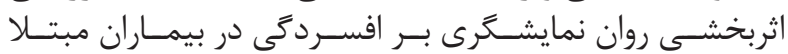

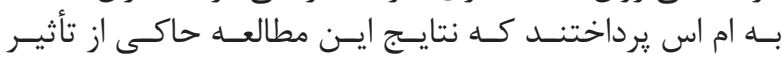

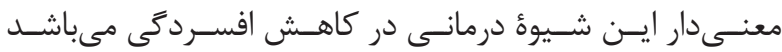

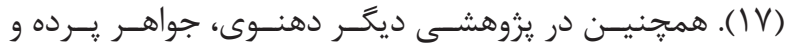

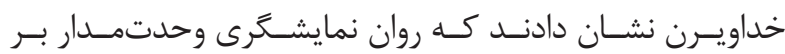

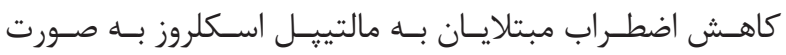

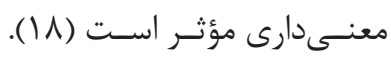

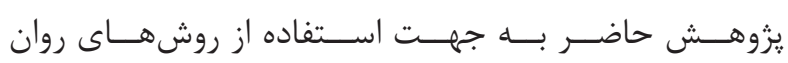

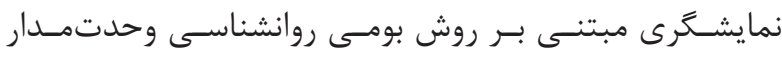

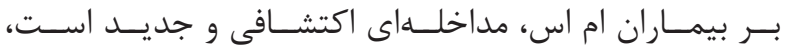

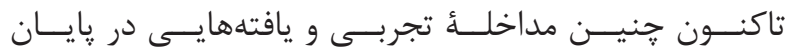

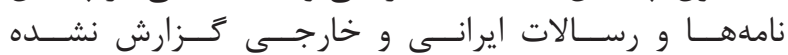

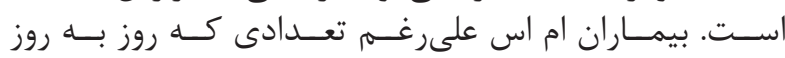

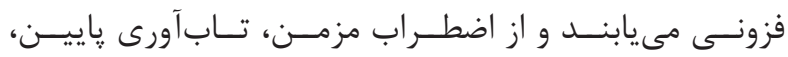

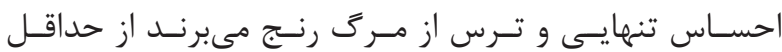

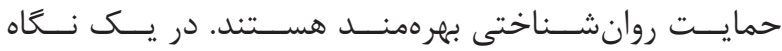

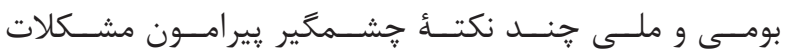

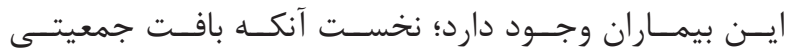

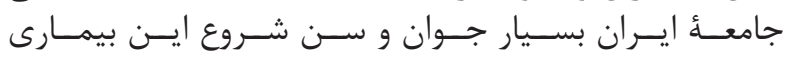

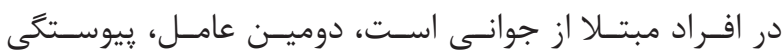

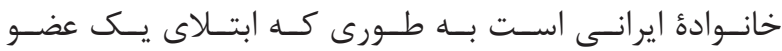

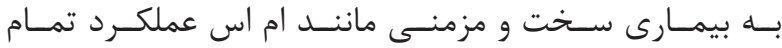

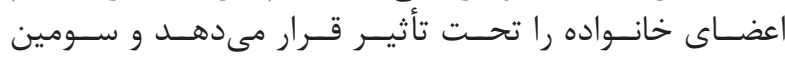

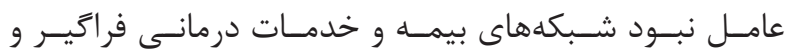

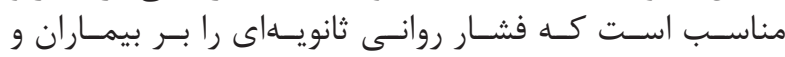

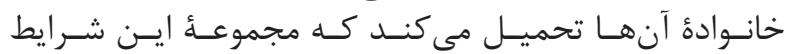

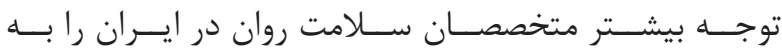

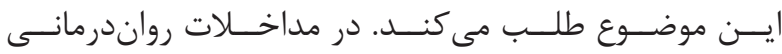

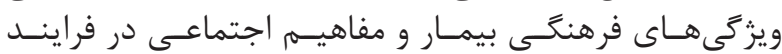

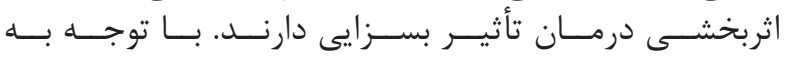

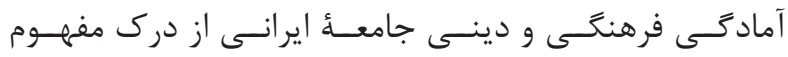

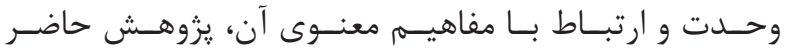

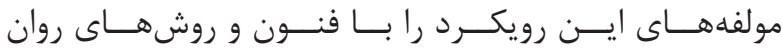

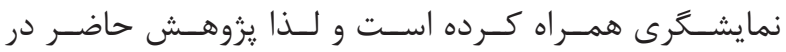

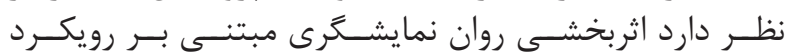

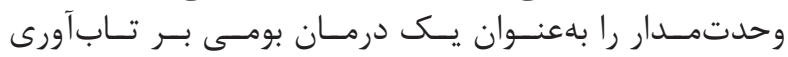

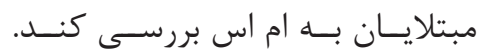




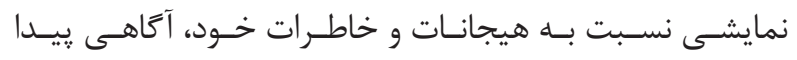

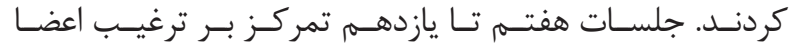

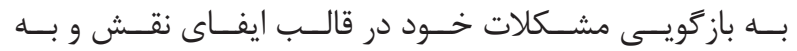

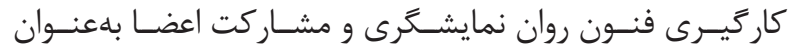

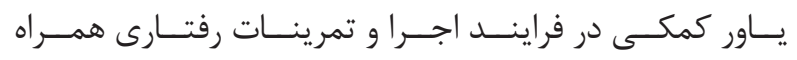

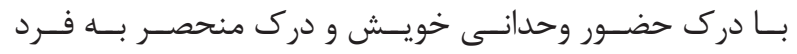

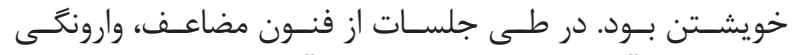

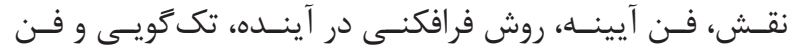

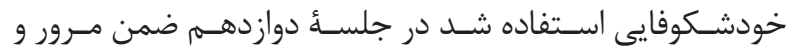

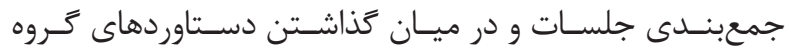

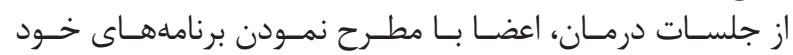

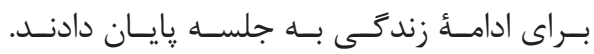

تجزيه و تحليل دادهها

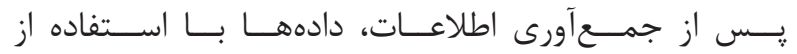

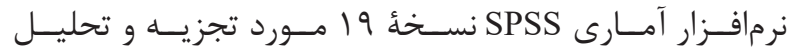

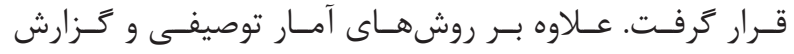

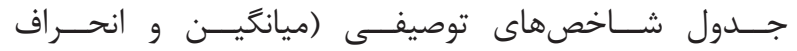

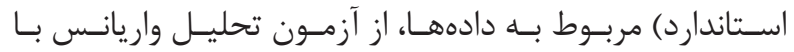

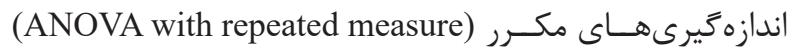
اسـتفاده شـد. هـ • • •

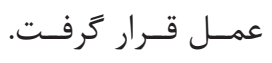

بافتهها

دادههـاى بــه دسـت آمــده بـا اسـتفاده از روشهــاى مناسـب

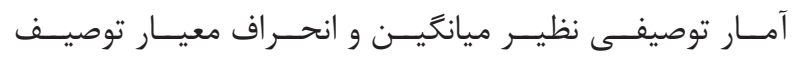

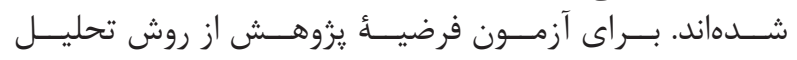

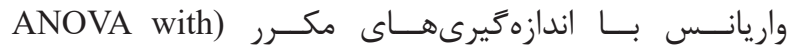
repeated measure

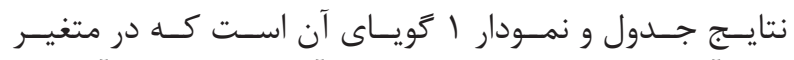

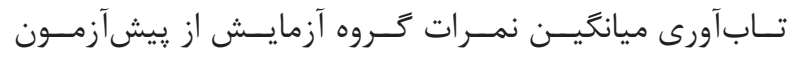

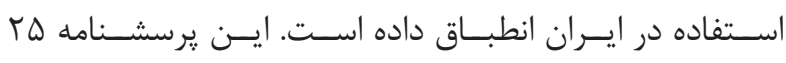

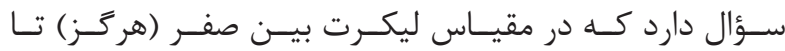

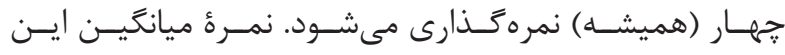

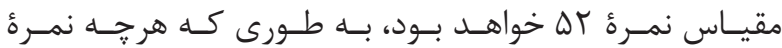

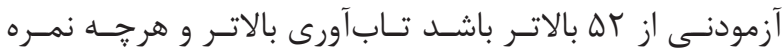

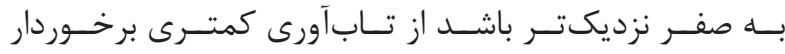

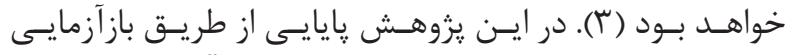

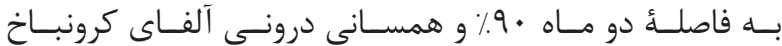

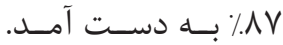
برنامة مداخلة د برمانى

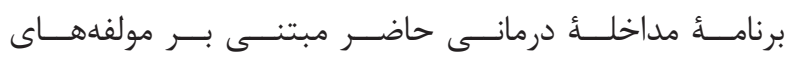

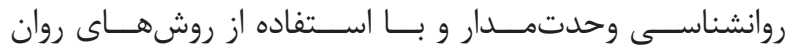

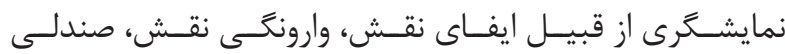

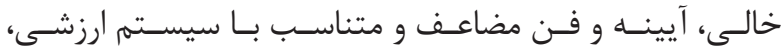

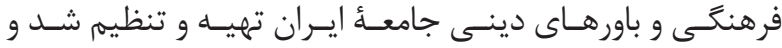

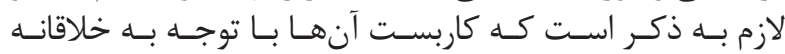

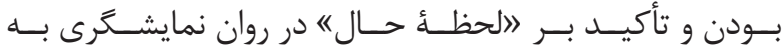

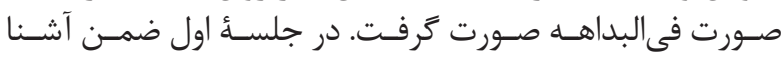

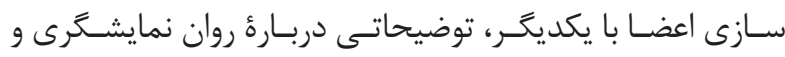

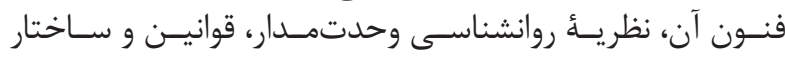

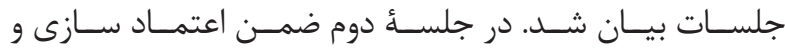

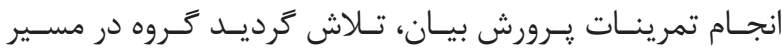

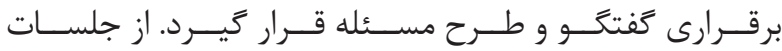

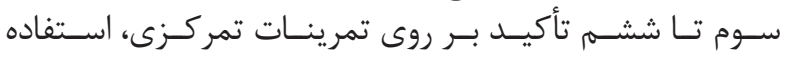

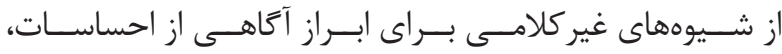

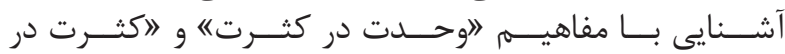

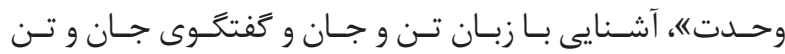

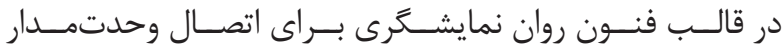

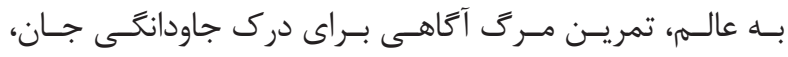

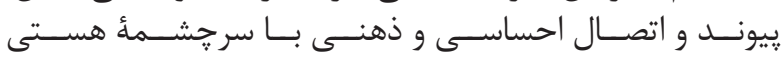

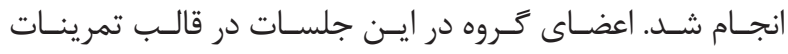

جدول ا- شاخصهاى توصيفى تابآورى به تفكيك دو كروه آزمايش و كنترل در سه مرحلة آزمايش.

\begin{tabular}{|c|c|c|c|c|c|c|}
\hline \multicolumn{2}{|c|}{ بيخيرى } & \multicolumn{2}{|c|}{ يس آزمون } & \multicolumn{2}{|c|}{ بيش آزمون } & \multirow{2}{*}{ كروه } \\
\hline SD & $\mathbf{M}$ & SD & $\mathbf{M}$ & SD & $\mathbf{M}$ & \\
\hline ה ATH & FF/F. & T/TAI & $F a / q$. & $F / l \Delta V$ & FN/A. & كنترل (+1+) \\
\hline FIFTV & $\Delta r / \mu$. & $F / r V V$ & $\Delta V / \Delta$. & F/ᄉ qF & $<q / \Lambda$. & آزمايش (+1n=1) \\
\hline
\end{tabular}

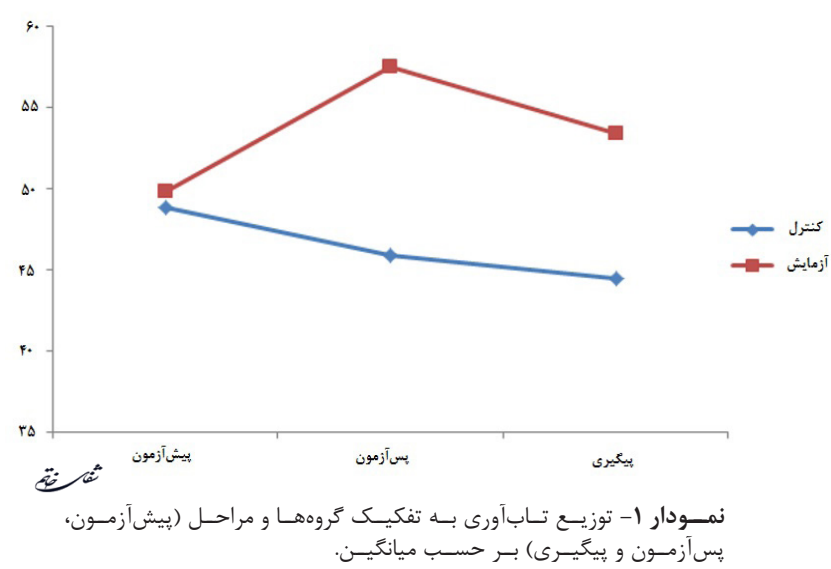




\begin{tabular}{|c|c|c|c|c|c|c|c|c|}
\hline 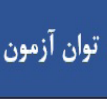 & اندازهُ اثر & $P$ & $\mathbf{F}$ & ميانكين مجذورات & df & مجذوروات & روند زمانى & منبع تغييرات \\
\hline \multirow[t]{2}{*}{$1 / \cdot \cdot$} & $\cdot / V A$ & $.1 . *^{*}$ & $s p / 4)$ & $\Delta \xi 1 / \Lambda$. & 1 & $\Delta \varphi 1 / \Lambda$. & \multirow{2}{*}{ 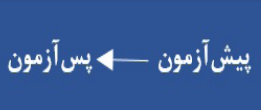 } & تعامل زمان و تروه \\
\hline & & & & $\Lambda / V Y$ & IN & $|Q Y| \cdot \cdot$ & & خطاى \\
\hline \multirow[t]{2}{*}{.149} & • & $.1 . . p * *$ & $1 \cdot / \Delta 9$ & $r \mu / \Lambda$. & 1 & $r \mu / \Lambda$. & \multirow{2}{*}{ يسآزمون - بـ ييكيرى } & تعامل زمان و گروه \\
\hline & & & & $r / 1 \Lambda$ & IN & $\Delta V / F$. & & خطاى \\
\hline.$/ 99$ & .194 & $\cdot 1 . * * * *$ & $r \cdot 119$ & $r r \cdot 1 . \cdot$ & 1 & $r r+1 . \cdot$ & \multirow{2}{*}{ بيش آزمون - ــ بيعيرى } & تعامل زمان و كَروه \\
\hline & & & & 1.19. & 11 & $19 \cdot / 1$. & & خطاى \\
\hline
\end{tabular}

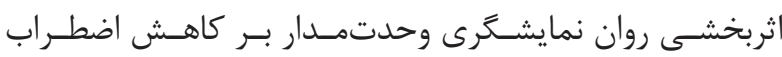

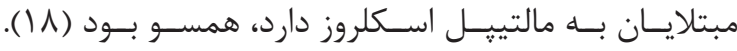

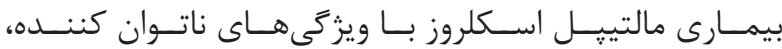

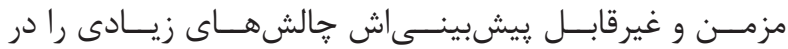

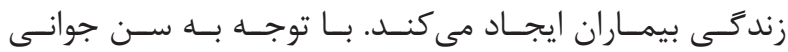

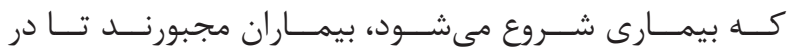

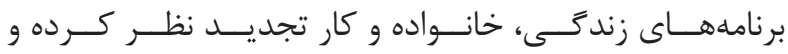

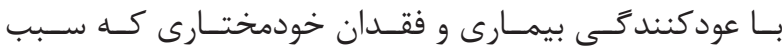

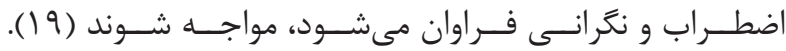

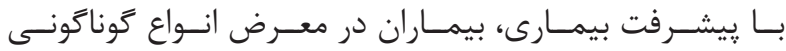

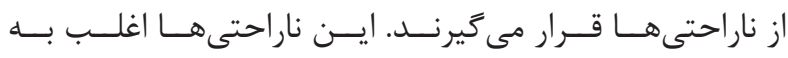

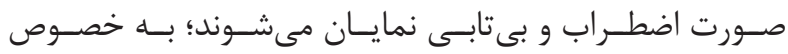

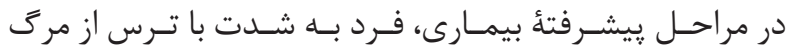

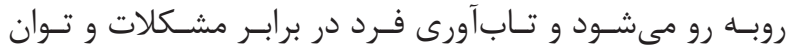

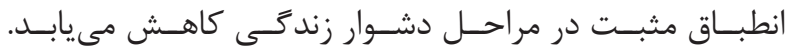

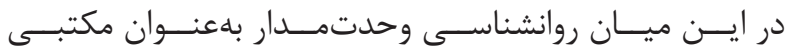

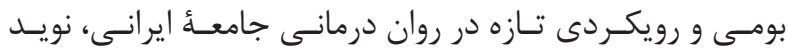

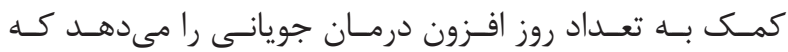

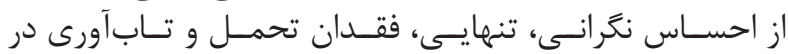

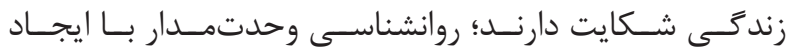

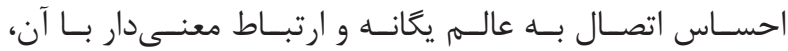

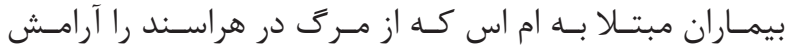

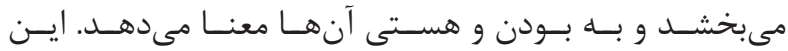

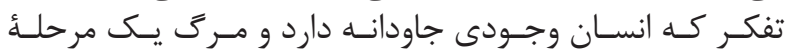

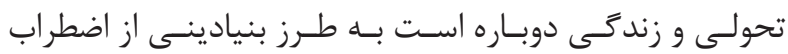

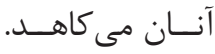

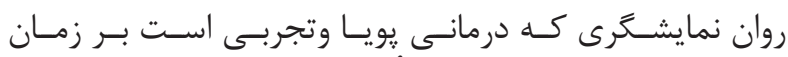

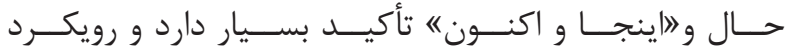

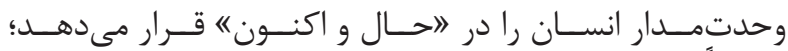

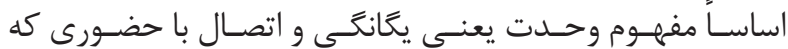

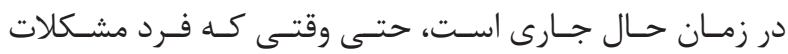

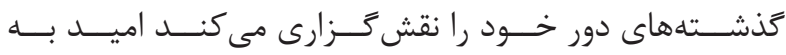

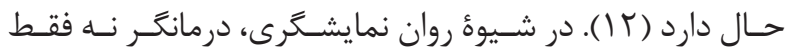

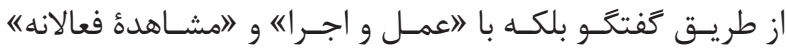

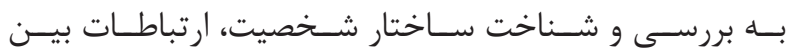

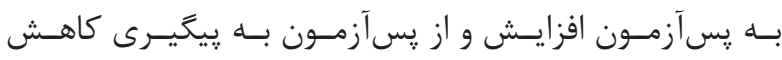

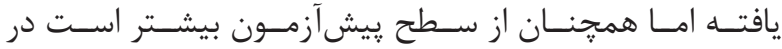

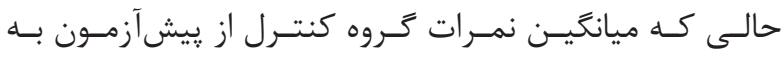

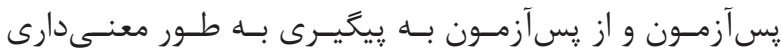

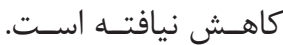

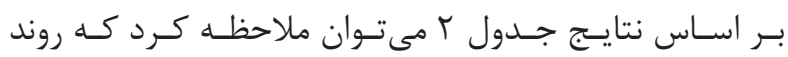

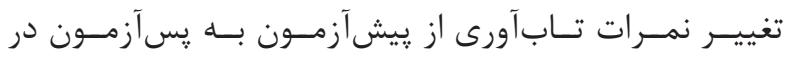

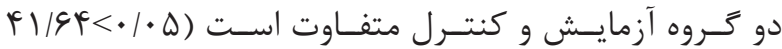

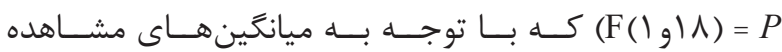

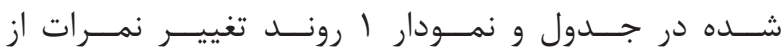

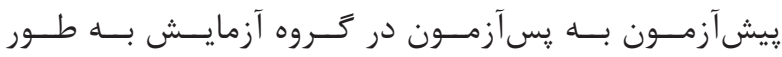

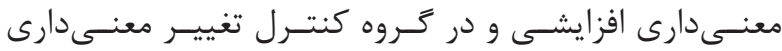

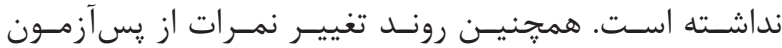

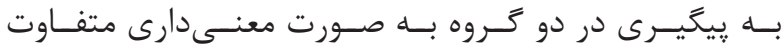

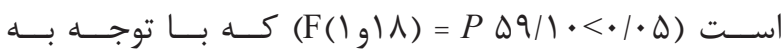

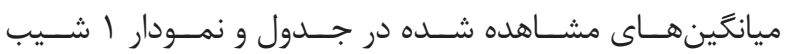

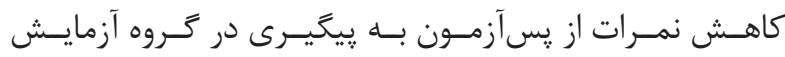

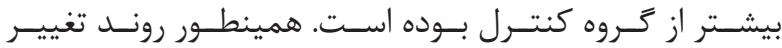

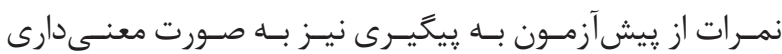

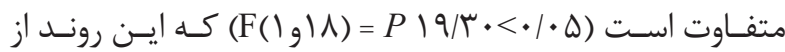

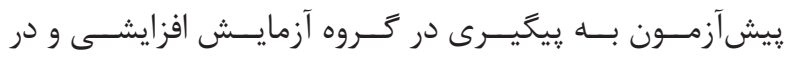

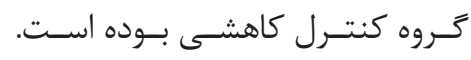

\section{بحث و نتيجهَ}

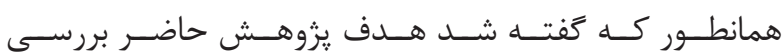

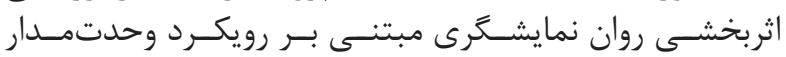

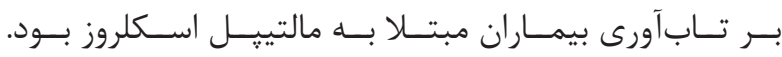

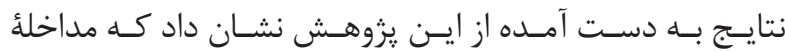

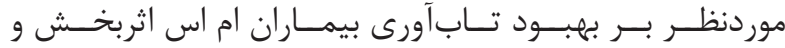

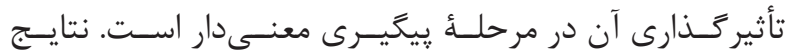

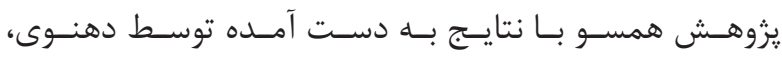

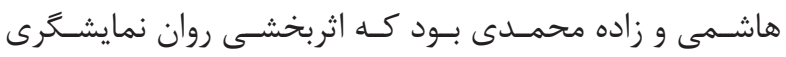

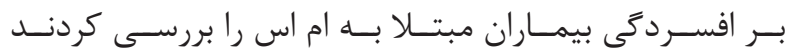

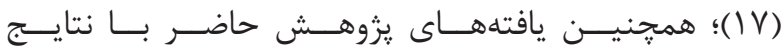

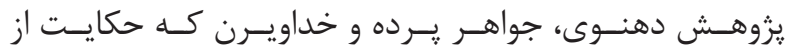




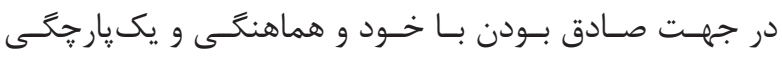

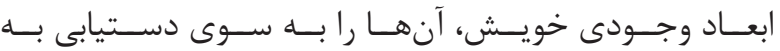

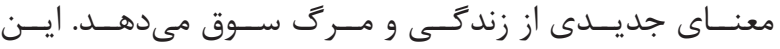

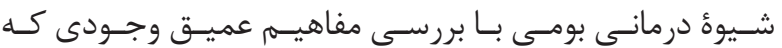

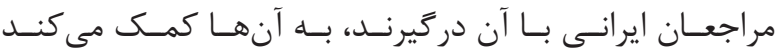

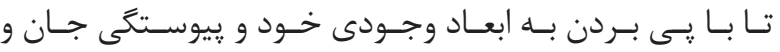

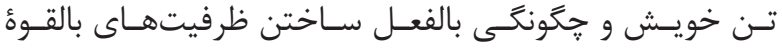

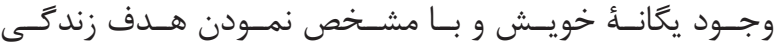

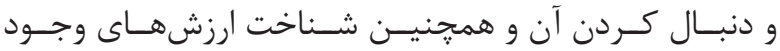

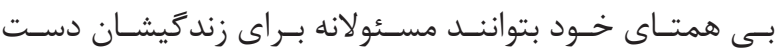

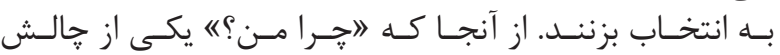

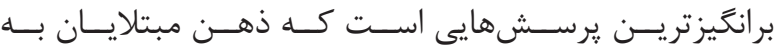

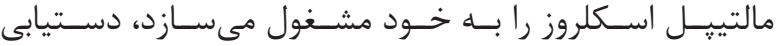

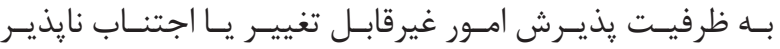

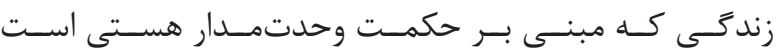

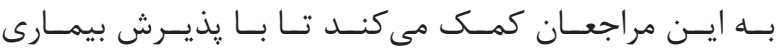

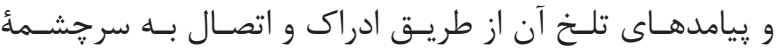

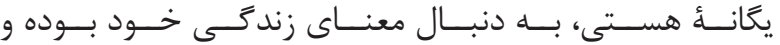

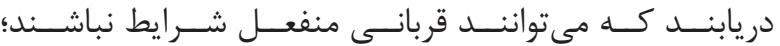

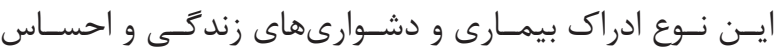

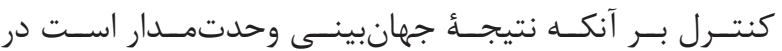

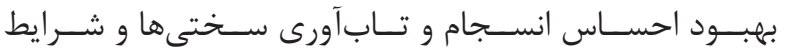

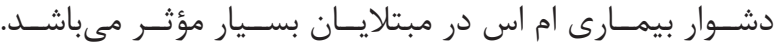

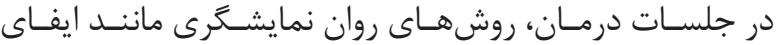

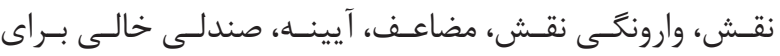

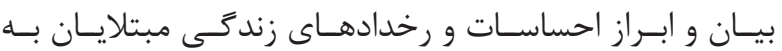

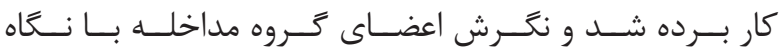

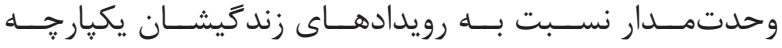

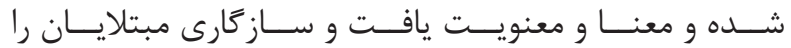

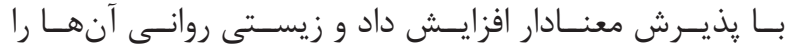
تقويست نمــود.

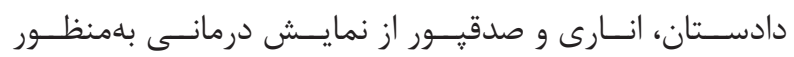

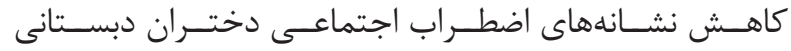

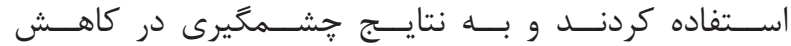

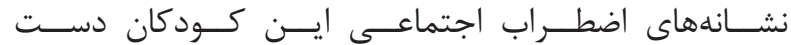

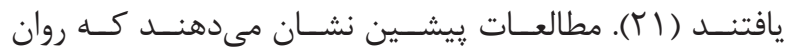

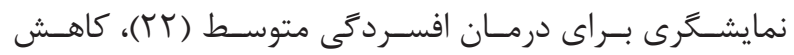

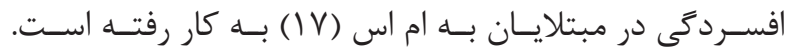

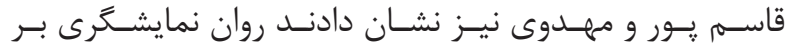

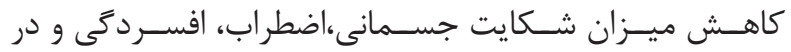

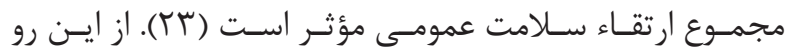

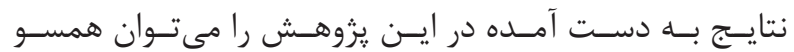

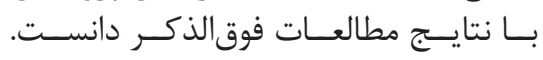

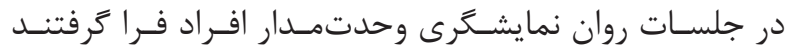

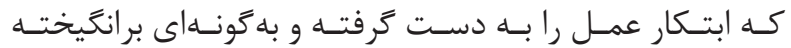

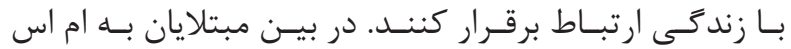

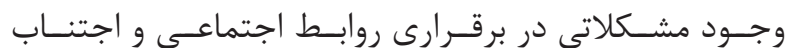

فــردى، تعارضـات درون و مسـائل هيجانسى بيمـار مى يــردازد

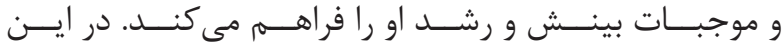

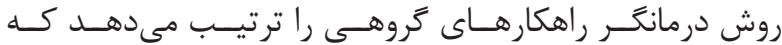

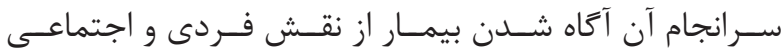

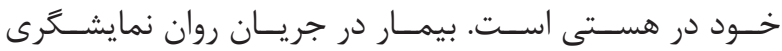

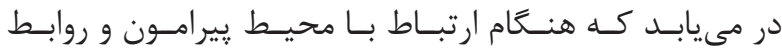

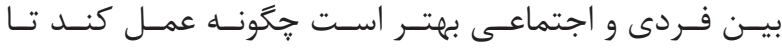

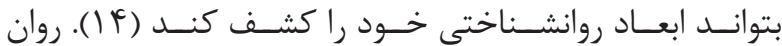

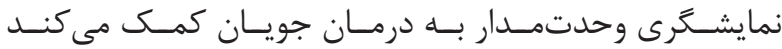

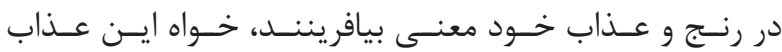

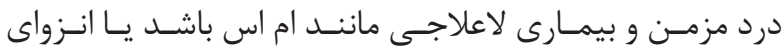

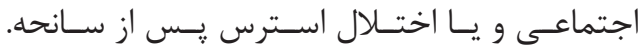

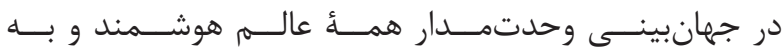

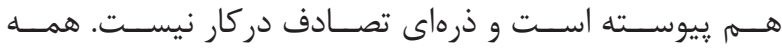

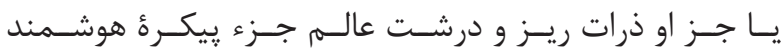

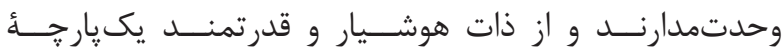

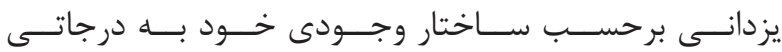

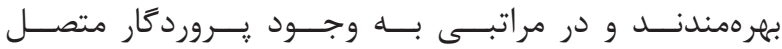

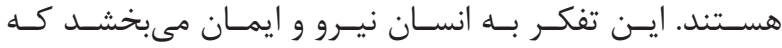

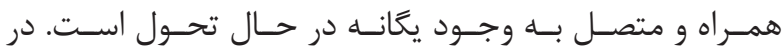

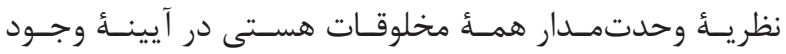

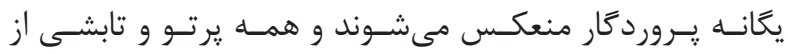

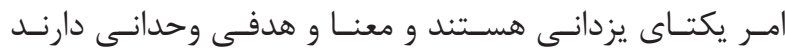

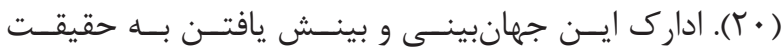

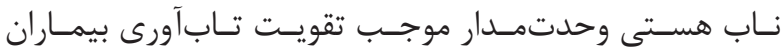

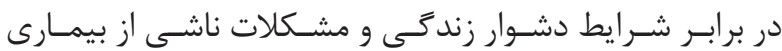

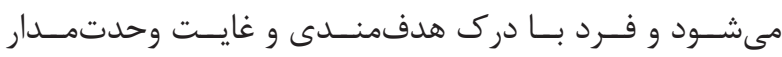

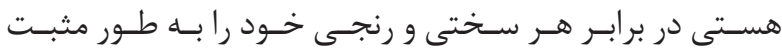

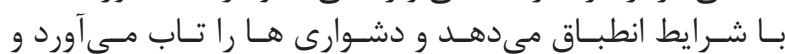

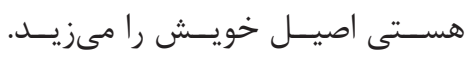

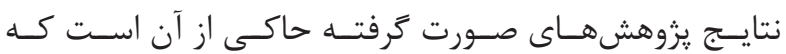

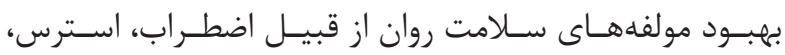

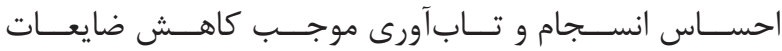

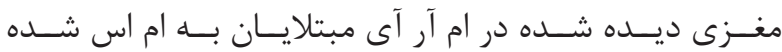

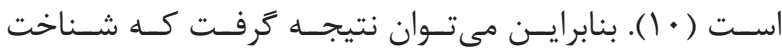

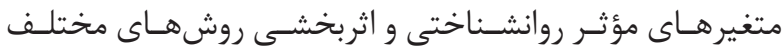

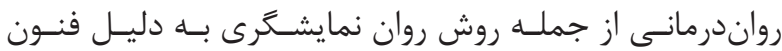

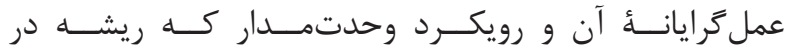

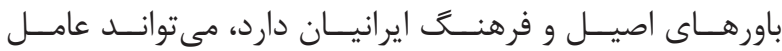

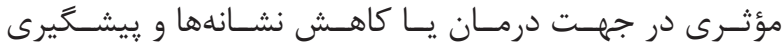

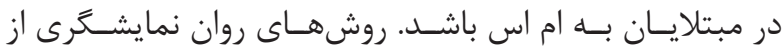

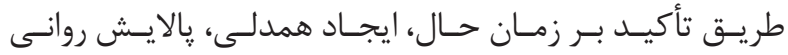

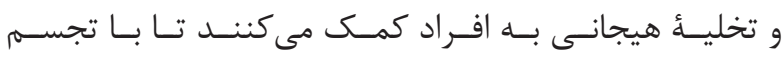

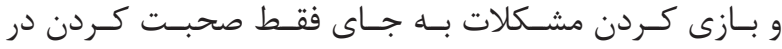

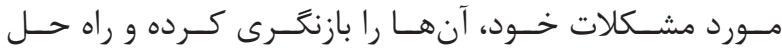

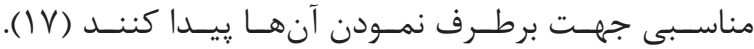

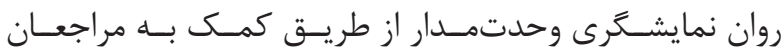




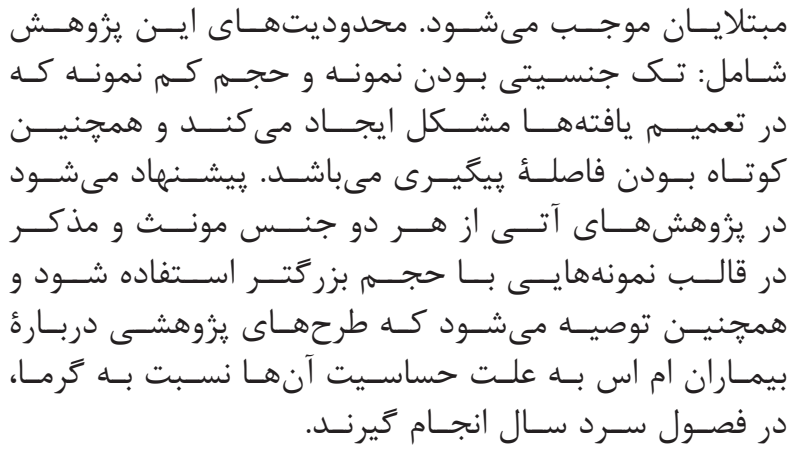

1. Halper J, Holland NJ. Multiple sclerosis: a self-care guide to wellness. Demos Medical Publishing; 2005. p. 736.

2. Frischer JM, Weigand SD, Guo Y, Kale N, Parisi JE, Pirko I, et al. Clinical and pathological insights into the dynamic nature of the white matter multiple sclerosis plaque. Ann Neurol. 2015; 78(5): 710-21.

3. Mirhashemi M, Najafi F. Efficacy of solution-centered therapy on resiliency and sense of coherence among patients with multiple sclerosis. Medical Sciences. 2014; 24(3): 175-81.

4. Dennison L, Moss-Morris R, Silber E, Galea I, Chalder T. Cognitive and behavioural correlates of different domains of psychological adjustment in early-stage multiple sclerosis. J Psychosom Res. 2010; 69(4): 353-61.

5. Janssens A, Doorn P, Boer J, Meche F, Passchier J, Hintzen R. Impact of recently diagnosed multiple sclerosis on quality of life, anxiety, depression and distress of patients and partners. Acta Neurol Scand. 2003; 108(6): 389-95.

6. Siegert R, Abernethy D. Depression in multiple sclerosis: a review. J Neurol Neurosurg Psychiatry. 2005; 76(4): 469-75.

7. Tabrizi N, Etemadifar M, Sharif A, Mirmehdi R. Multiple sclerosis. Tehran: Ghaed Publication; 2012.

8. Luthar SS, Cicchetti D. The construct of resilience: Implications for interventions and social policies. Dev Psychopathol. 2000; 12(4): 857-85.

9. Mandel G, Mullet, E, Brown G. Cultivating resiliency a guide for parent and school personnel. http://www. scholastic. com: scholastic press. 2006.

10. Rattue P. Stress reduction therapy could significantly help MS patients. Medical News Today. http://www.

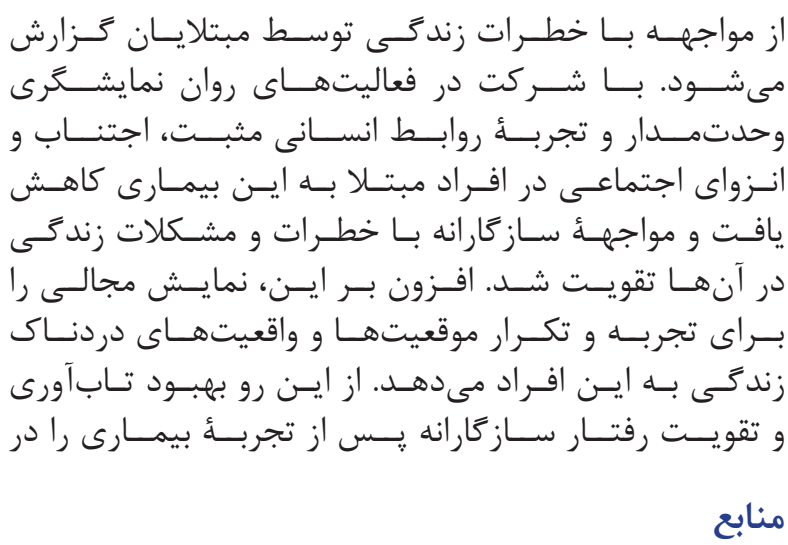

MedicalNewsToday.Com. 2012.

11. Dayapoğlu N, Tan M. Evaluation of the effect of progressive relaxation exercises on fatigue and sleep quality in patients with multiple sclerosis. J Altern Complement Med. 2012; 18(10): 983-7.

12. Mohammadi Z. Unity-oriented psychology. Iran: Qatreh Publications; 2011.

13. Leveton E. A clinicians guide to psychodrama. $3^{\text {rd }} \mathrm{ed}$. Springer Publication; 2001.

14. Blatner A. Acting-in: practical applications of psychodramatic methods. Tehran: Roshd Publication; 2005.

15. Kooraki M, Yazdkhasti F, Ebrahimi A, Oreizi H. Effectiveness of psychodrama in improving social skills and reducing internet addiction in female students. Iranian Journal of Psychiatry and Clinical Psychology. 2012; 17(4): 279-88.

16. Dehnavi S, Ayazi Nofuti M, Bajelan M. The effectiveness of psychodrama in relapse prevention and reducing depression among opiate-dependent men. Addiction Quarterly Journal of Drug Abuse. 2015: 34(9): 87-94.

17. Dehnavi S, Hashemi S-F, Zadeh-Mohammadi A. The effectiveness of psychodrama on reducing depression among multiple sclerosis patients. IJBS. 2016; 10(1): 32-5.

18. Dehnavi S, Javaher Parde S, KhodaViren H, Bajalan M. Effectiveness of psychodrama on anxiety of multiple sclerosis patients. Mediterranean Journal of Social Sciences. 2016; 7(4) S1: 150-4.

19. Landoni MG, Giordano MT, Guidetti GP. Group psychotherapy experiences for people with multiple sclerosis and psychological support for families. Journal of Neurovirology. 2000; 6(2): S168-71. 


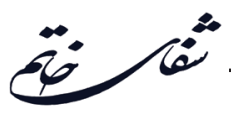

20. Mohammadi Z. Unity-oriented worldview. Iran: Qatreh Publications; 2014.

21. Dadsetan P, Anari A, Saleh Sedghpour B. Social anxiety disorders and drama-therapy. Journal of Iranian Psychologists. 2008; 14(4): 115-23.
22. Hamamci Z. Integrating psychodrama and cognitive behavioral therapy to treat moderate depression. The Arts in Psychotherapy. 2006; 33(3): 199-207.

23. Ghasempoor A, Mahdavi A. Survey effect of psychodrama on promoting public health. Journal of Rahpooye of Art. 2010; 3(13): 11-20. 